\title{
Health-related quality of life in Polish adolescents with Hymenoptera venom allergy treated with venom immunotherapy
}

\author{
Ewa Cichocka-Jarosz $z^{1}$, Piotr Brzyski², Ewa Świebocka ${ }^{3}$, Joanna Lange ${ }^{4}$, Beata Tobiasz-Adamczyk²,

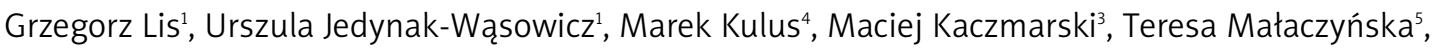 \\ Barbara Klajna-Kraluk, Anna Bręborowicz ${ }^{6}$, Zdzisława Kycler ${ }^{6}$, Jacek J. Pietrzyk ${ }^{1}$
}

\author{
${ }^{1}$ Chair of Pediatrics, Department of Pediatrics, Jagiellonian University Medical College, \\ Krakow, Poland \\ 2Department of Medical Sociology, Chair of Epidemiology and Preventive Medicine, \\ Jagiellonian University Medical College, Krakow, Poland \\ 3Department of Pediatrics, Children's Gastroenterology and Allergology, \\ Medical University of Bialystok, Poland \\ ${ }^{4}$ Department of Pediatric Pulmonology and Allergy, Warsaw Medical University, \\ Poland \\ ${ }^{5}$ Regional Center of Allergology, Pulmonology and Cystic Fibrosis, Gdansk, Poland \\ ${ }^{6}$ Department of Pneumonology, Allergology and Clinical Immunology, \\ Poznan University of Medical Sciences, Poland
}

Submitted: 29 February 2012

Accepted: 20 May 2012

Arch Med Sci 2012; 8, 6: 1076-1082

DOI: 10.5114/aoms.2012.32419

Copyright @ 2012 Termedia \& Banach

\begin{abstract}
Introduction: Venom allergy, though rare, may seriously influence health-related quality of life (HRQoL). There is a paucity of research on HRQoL of adolescents and young adults with Hymenoptera venom allergy. The aim was to assess the level of HRQoL and to evaluate its independent predictors in Polish adolescents and young adults treated with venom immunotherapy.

Material and methods: A multicenter cross-sectional study based on the Vespid Allergy Quality of Life Questionnaire (VQLQ) adapted for Polish adolescents was used. The study sample included 87 patients (14-21 years) studied at different stages of venom immunotherapy (VIT). Statistical analysis was done with multivariate linear regression.

Results: Anxiety level was higher in patients with $4^{\text {th }}$ grade of Mueller's classification (anaphylactic shock) than in those with $3^{\text {rd }}$ grade $(B=0.84,95 \% \mathrm{Cl}=$ $0.07-1.61, p=0.03)$. Caution increased along with an increase of anxiety of adolescents treated with VIT $(B=0.54,95 \% \mathrm{Cl}=0.39-0.68, p<0.01)$. Level of limitations increased with increasing caution of adolescents $(B=0.63,95 \% \mathrm{Cl}=$ $0.35-0.91, p<0.01)$. Discomfort increased along with a rise of caution of patients $(B=0.38,95 \% \mathrm{Cl}=0.22-0.55, p<0.01)$. Similarly, it increased with an increase of their feeling of limitations $(B=0.37,95 \% \mathrm{Cl}=0.23-0.51, p<0.01)$. The level of discomfort in adolescents treated with VIT was lower in those who were treated with conventional protocol in comparison to those treated with rush or ultrarush ones $(B=-0.47,95 \% \mathrm{Cl}=-0.90--0.03, p=0.04)$.

Conclusions: Severity of anaphylactic reaction is an independent determinant of anxiety level in adolescents treated with VIT. The VIT protocol affects HRQoL of treated patients.
\end{abstract}

Key words: Vespid Allergy Quality of Life Questionnaire, insect allergy, disease burden.

\author{
Corresponding author: \\ Ewa Cichocka-Jarosz MD, PhD \\ Chair of Pediatrics \\ Department of Pediatrics \\ Jagiellonian University \\ Medical College \\ 265 Wielicka St \\ 30-663 Krakow, Poland \\ Phone: 481265820 11, \\ ext. 1655 \\ Fax: 48126584446 \\ E-mail:mijarosz@cyfronet.pl
}




\section{Introduction}

Hymenoptera venom allergy (HVA), though rare, is a potentially life-threatening disorder, which requires a high level approach, appropriate to allergy intensity. Mueller's four-grade classification of systemic reactions to insect stings includes general urticaria (grade I), angioedema (grade II), stridor or wheezing (grade III) and anaphylactic shock (grade IV). Subcutaneous injections of venom immunotherapy (VIT) are a treatment of choice for patients with grades III and IV. In patients with grades I and II, additional factors, such as high exposure to venoms or impaired health-related quality of life (HRQoL) due to venom allergy, are taken into consideration before making a decision of VIT introduction. The VIT procedure consists of two phases: the incremental dose phase and the maintenance dose phase. The incremental dose phase employs different protocols, which allow for achieving the maintenance dose phase from within 12 weeks (conventional) to a few days (rush) or a few hours (ultra-rush). The maintenance dose is given every 4-6 weeks usually for 3-5 years [1, 2].

The impact of insect venom allergy on HRQoL may be associated with several social restrictions and limitations in everyday activities of adolescents. These social limitations influenced by health conditions also trigger changes in psychological wellbeing: fear, anxiety, uncertainty and loss of safety can be expected as a risk of insect venom allergy consequences. The extent to which psychosocial consequences of allergy are a burden to adolescent patients may be influenced by sociodemographic (e.g. gender, age or place of residence) or medical (e.g. grade of anaphylactic reaction or VIT duration) factors (Figure 1).

Several instruments have been developed to measure HRQOL in children and adolescents suffering from different health conditions including allergic diseases [3-9]. However, the spectrum of HRQoL scales addressed to adolescents is still outnumbered by scales addressed to adult patients [10-13].

To assess HRQoL in adult patients with yellow jacket (Vespula) allergy, the Vespid Allergy Quality of Life Questionnaire (VQLQ) was developed [14]. The presented results come from the first multicenter study conducted with adaptation of VQLQ for Polish adolescents treated with VIT [15].

The aim of the study was to assess HRQoL in adolescents and young adults with yellow jacket or honey bee allergy, treated with VIT in Poland, and to evaluate the impact of demographic, social and medical factors on patients' HRQoL.

\section{Material and methods}

\section{Patient recruitment}

The study sample consisted of 87 adolescents and young adults, aged from 14 to 21 years (mean age: 17.0 years, SD 2.1 years), who were treated with VIT in September of 2008 in five clinical centers in Poland, covering almost the whole population at this age treated with VIT in Poland at that time. Patients were included in the study after completion of the written consent form by themselves and by one of their parents. The study was approved by the Ethics Committee of Jagiellonian University (number of decision: KBET no. 67/L/2007). The characteristics of the group are listed in Table I.

\section{Measures}

Face-to-face interviews were conducted using a structured questionnaire containing demographic data, and the adaptation of VQLQ for Polish adolescents. Vespid Allergy Quality of Life Questionnaire is a tool consisting of 14 items concerning

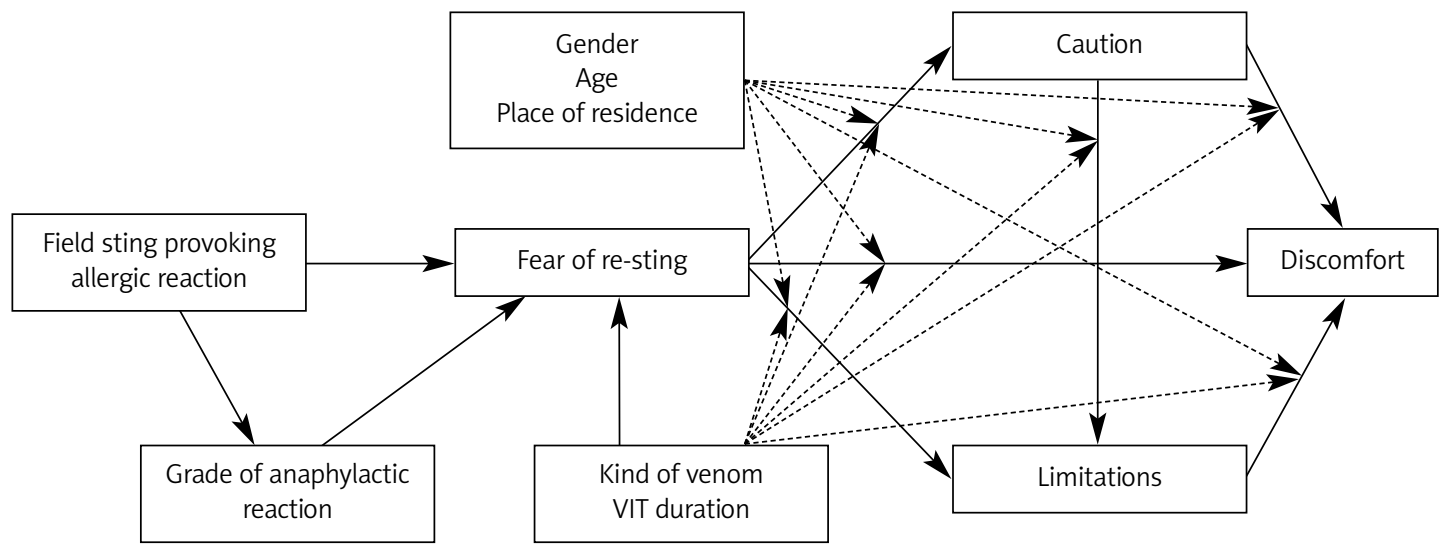

Figure 1. Model of the study

Solid lines represent both 1. hypothetical medical factors which may influence the level of $H R Q O L$ and 2. the hypothesized relationship between particular dimensions of HRQOL. Dashed lines represent the hypothetical impact of confounding variables on the level of particular dimensions of HRQOL. 
the following: anxiety of being stung by insects, behaviors to avoid further stinging (caution), limitations in outdoor activities during summer and feeling of being troubled by looking for insects in different settings (discomfort) [15]. Every item has a 7-point Likert response scale. Scores of particular subscales were computed as the algebraic mean of its items, resulting in scores ranging from 1 (reflecting the lowest level of measured latent variables) to 7 (reflecting the highest one: highest anxiety, highest caution, etc).

Gender of patients, age and place of residence were treated as potential socio-demographic predictors of HRQoL. Due to a relatively small number of girls in the study sample, and different patterns of relationship between dimensions of quality of life and age observed in boys, these two sociodemographic variables were combined into one crossproduct variable indicating differences between younger boys (aged 17 years and less) and older boys (aged 18 years and more) in relation to girls (irrespectively of their age), treated as the reference category for this variable. Place of residence was used as a binary variable differentiating those living in villages from those living in towns as the reference category.

Medical factors, such as kind of culprit insect, incremental dose protocol and grade of clinical reaction after being stung, were used as dummy variables. Namely the treatment against wasp venom,

Table I. Medical characteristics of the group

\begin{tabular}{|c|c|c|}
\hline Parameter & $N$ & $\%$ \\
\hline \multicolumn{3}{|l|}{ Gender and age } \\
\hline Girls & 15 & 17.2 \\
\hline Younger boys & 40 & 46.0 \\
\hline Older boys & 32 & 36.8 \\
\hline \multicolumn{3}{|c|}{ Place of residence } \\
\hline Town & 32 & 36.8 \\
\hline Village & 55 & 63.2 \\
\hline \multicolumn{3}{|c|}{ Immunotherapy against } \\
\hline Wasp & 40 & 46.0 \\
\hline Bee & 43 & 49.4 \\
\hline Both & 4 & 4.6 \\
\hline \multicolumn{3}{|c|}{ Mueller's grade before VIT } \\
\hline 2 & 9 & 10.3 \\
\hline 3 & 54 & 62.1 \\
\hline 4 & 24 & 27.6 \\
\hline \multicolumn{3}{|l|}{ Protocol } \\
\hline Ultra-rush & 16 & 18.4 \\
\hline Rush & 46 & 52.9 \\
\hline Conventional & 25 & 28.7 \\
\hline
\end{tabular}
(83\%), and mostly residents of rural areas (63\%). Mean age of adolescents was 17.0 years (SD 2.1 years). The number of patients allergic to either bee venom or yellow jacket venom was almost equal. The majority of patients were treated with the rush protocol and most of them reported $3^{\text {rd }}$ grade of Mueller's classification before VIT (Table I).

The patients were studied at different periods of VIT course, with a median of 3 years of treatment $(\mathrm{Q} 1=1, \mathrm{Q} 3=5)$. The median age of the first sting was 10 years $(\mathrm{Q} 1=7, \mathrm{Q} 3=12)$ while the median age at beginning VIT was $13(\mathrm{Q} 1=12, \mathrm{Q} 3=15)$. The median number of stings before VIT was $3(\mathrm{Q} 1=1, \mathrm{Q} 3=7)$.

\section{Univariate analysis}

Among the analyzed dimensions of HRQoL, the highest median was observed for the anxiety and caution subscales, the lowest for discomfort (Table II).

Older boys had significantly lower medians of anxiety and feeling of limitations than younger ones and girls (irrespectively of their age). A similar pattern was noted in caution. Significant differences in anxiety were observed in relation to severity of anaphylactic reaction: the median values 
Table II. Average levels of particular dimensions of quality of life

\begin{tabular}{|lccc|}
\hline Variable & Median & 1 $^{\text {st }}$ Quartile & $3^{\text {rd }}$ Quartile \\
\hline Anxiety & 3.5 & 2.5 & 5.0 \\
\hline Caution & 3.5 & 2.8 & 4.5 \\
\hline Limitations & 3.0 & 1.0 & 4.0 \\
\hline Discomfort & 2.2 & 1.3 & 3.0 \\
\hline
\end{tabular}

Table III. Differences in quality of life level due to analyzed factors

\begin{tabular}{|c|c|c|c|c|}
\hline Variable & Median & $\begin{array}{c}1^{\text {st }} \\
\text { Quartile }\end{array}$ & $\begin{array}{c}3^{\text {rd }} \\
\text { Quartile }\end{array}$ & $\begin{array}{c}\text { Value } \\
\text { of } p\end{array}$ \\
\hline \multicolumn{5}{|l|}{ Anxiety } \\
\hline \multicolumn{5}{|l|}{ Gender and age: } \\
\hline Girls & 4.0 & 3.5 & 5.0 & \multirow[t]{3}{*}{0.02} \\
\hline Younger boys & 4.0 & 2.5 & 5.9 & \\
\hline Older boys & 3.0 & 2.0 & 4.0 & \\
\hline \multicolumn{5}{|c|}{ Mueller's grade before VIT: } \\
\hline 2 & 3.0 & 1.5 & 4.3 & \multirow[t]{2}{*}{0.03} \\
\hline 3 & 3.5 & 2.5 & 4.6 & \\
\hline 4 & 4.0 & 3.1 & 5.9 & \\
\hline \multicolumn{5}{|l|}{ Caution } \\
\hline \multicolumn{5}{|l|}{ Gender and age: } \\
\hline Girls & 3.5 & 3.0 & 4.5 & \multirow[t]{3}{*}{0.05} \\
\hline Younger boys & 4.0 & 3.0 & 5.4 & \\
\hline Older boys & 3.0 & 2.3 & 3.7 & \\
\hline \multicolumn{5}{|c|}{ Place of residence: } \\
\hline Town & 3.8 & 3.1 & 5.3 & \multirow[t]{2}{*}{0.05} \\
\hline Village & 3.3 & 2.5 & 4.5 & \\
\hline \multicolumn{5}{|l|}{ Limitations } \\
\hline \multicolumn{5}{|l|}{ Gender and age: } \\
\hline Girls & 3.0 & 2.0 & 5.0 & \multirow[t]{3}{*}{0.01} \\
\hline Younger boys & 3.0 & 1.0 & 5.0 & \\
\hline Older boys & 2.0 & 1.0 & 3.0 & \\
\hline
\end{tabular}

of anxiety increased along with Mueller's grade. Higher caution was reported by residents of urban areas (Table III).

\section{Multivariate analysis}

The multivariate linear regression model showed that anxiety increased along with a rise of Mueller's grade: in patients with $4^{\text {th }}$ grade (life-threatening reaction), it was significantly higher than in other patients. The level of anxiety of adolescents treated with VIT was lower in older boys than in girls (irrespectively of their age); however, this difference was of borderline significance (Table IV).
Table IV. Independent determinants of anxiety according to multivariate linear regression model

\begin{tabular}{|lccccc|}
\hline Variable & \multicolumn{3}{c}{$B$} & Value of $p$ & \multicolumn{2}{c|}{$95 \% \mathrm{Cl}$ for $B$} \\
\hline Younger boys vs. girls & -0.44 & 0.37 & -1.40 & 0.53 \\
\hline Older boys vs. girls & -0.97 & 0.08 & -2.05 & 0.10 \\
\hline $\begin{array}{l}\text { Mueller's grade: } \\
\text { 2 vs. 3 (ref.) }\end{array}$ & -0.31 & 0.62 & -1.51 & 0.90 \\
\hline $\begin{array}{l}\text { Mueller's grade: } \\
\text { 4 vs. 3 (ref.) }\end{array}$ & 0.84 & 0.03 & 0.07 & 1.61 \\
\hline \begin{tabular}{l} 
VIT duration (cont.) \\
\hline
\end{tabular} & -0.01 & 0.09 & -0.03 & 0.00 \\
\hline
\end{tabular}

Table V. Independent determinants of limitations level according to multivariate linear regression model

\begin{tabular}{|lccccc|}
\hline Variable & $B$ & Value of $p$ & \multicolumn{2}{c|}{$95 \% \mathrm{Cl}$ for $B$} \\
\hline Younger boys vs. girls & -0.31 & 0.60 & -1.47 & 0.86 \\
\hline Older boys vs. girls & -1.57 & 0.03 & -2.98 & -0.16 \\
\hline $\begin{array}{l}\text { Place of residence: rural } \\
\text { vs. urban areas (ref.) }\end{array}$ & 0.33 & 0.64 & -1.06 & 1.72 \\
\hline Anxiety & 0.17 & 0.15 & -0.06 & 0.40 \\
\hline Caution & 0.63 & 0.00 & 0.35 & 0.91 \\
\hline $\begin{array}{l}\text { Younger boys place } \\
\text { of residence }\end{array}$ & 0.50 & 0.54 & -1.12 & 2.11 \\
\hline $\begin{array}{l}\text { Older boys place } \\
\text { of residence }\end{array}$ & 1.41 & 0.12 & -0.37 & 3.19 \\
\hline
\end{tabular}

Caution of adolescents treated with VIT increased along with an increase of their anxiety $(B=0.54,95 \% \mathrm{Cl}=0.39-0.68, p<0.01)$. The difference in caution between residents of villages and residents of towns was of borderline significance $(B=-0.44,95 \% \mathrm{Cl}=0.93-0.04, p=0.07)$.

Level of limitations increased along with caution of adolescents, and it was significantly lower in older boys in comparison to girls, both living in towns; however, differences between boys regarding place of residence were not significant (Table $\mathrm{V}$ ).

Discomfort increased along with a rise of caution and the feeling of limitations $(B=0.38,95 \% \mathrm{Cl}$ $=0.22-0.55, p<0.01$ and $B=0.37,95 \% \mathrm{Cl}=0.23$ $0.51, p<0.01$, respectively). The level of discomfort was lower in those who were treated with the conventional protocol in comparison to those treated with rush or ultra-rush ones $(B=-0.47,95 \% \mathrm{Cl}=$ $-0.90-0.03, p=0.04$ ).

\section{Discussion}

Health-related quality of life in HVA patients is poorly evaluated. Until the development of VQLQ by Oude-Elberink, which was the first measurement tool specific for vespid venom allergic adults, other attempts had a rather exploratory character $[1,14,16,17]$. The present study is the first part of multicenter research examining at the same time 
point HRQoL in almost the entire population of VITtreated Polish adolescents. The studies based on VQLQ were performed in the Netherlands and English-speaking countries; however, up till now VQLQ has been applied as a unidimensional index and verification of its multidimensionality is not known to the authors $[14,18]$.

The median level of QoL reported by Polish adolescents ranges from 2.2 to 3.5. This suggests that HRQoL of Polish adolescents, regardless of the kind of insect causing sensitization, is less impaired than that of Dutch adults with wasp venom allergy. Oude-Elberink reports mean scores of the VQLQ scale of 4.3 in pre-treatment measurements and 4.6 after 1 year of treatment [18]. In another paper, the same author reported scores of 3.3 in pre-treatment measurements vs. 4.4 after 1 year of treatment in patients who were randomly selected for treatment with VIT or Epipen adrenaline in autosyringe, reporting improvement in HRQoL in both groups. A somewhat different situation was reported by patients who decided about VIT treatment themselves: they reported improvement from 2.8 to 4.3 after 1 year of treatment [19].

The results of factor analysis suggesting multidimensionality of the scale $[15,20]$ are supported by the results of linear regression multivariate models presented in this study, which resembles the results obtained in a study using adaptation of VQLQ for Polish children [21]. The multivariate models revealed social and medical factors influencing the scores of analyzed dimensions of HRQoL in adolescents treated with VIT: significant associations were revealed between Mueller's grade of anaphylactic reaction and anxiety, place of residence and limitations, as well as between the protocol of VIT and level of discomfort.

We observed a deteriorated level of HRQoL, reflected in an increase of anxiety along with a rise of Mueller's grade, reported in the sting history before treatment. This is not consistent with the Dutch results, which revealed no correlation of the VQLQ score with the degree of systemic reaction [18]. It is possible that this effect exists, but might have been suppressed by "including" anxiety level in the total score of VQLQ.

An observed significant positive relationship between anxiety and caution means that higher anxiety of being stung results in higher care to avoid it. This result is consistent with the observation made in samples of Polish children with HVA and their parents [21]. We also noted a lower level of caution in adolescents from rural areas. Perhaps they were more accustomed to the presence of Hymenoptera, as one third of patients living in rural areas are bee-keepers family members.

An observed increase of feeling of limitations during summer along with a rise of caution indicates that behaviors aimed at avoiding being stung result in abandonment of different activities. Studies conducted in Polish children with HVA did not confirm a similar relationship, whereas the same analysis conducted in their parents showed a decrease in limitations imposed on their children along with an increase of parental anxiety, which may suggest that placing limitations on one's children is a consequence of felt anxiety and the most obvious way to decrease that feeling [21].

We observed a significant difference in the level of limitations between older boys (aged 18 years and over) and girls, both living in towns, but such differences did not exist between adolescents living in rural areas. It seems to be a combined effect of differences regarding the level of risk of being stung and differences regarding the social context of changes related to achieving the age of maturity.

The noted significant positive influence of both caution and limitations on discomfort means that a higher feeling of discomfort is related to avoidance behaviors, and results in a higher feeling of limitations. A similar impact of caution on the level of discomfort was observed in parents of children treated with VIT in Poland [21].

We also observed a lower level of discomfort in patients treated with a longer lasting conventional protocol. From the medical point of view, shorter protocols, though requiring a higher number of subcutaneous injections in a short time, are safe, and they allow immunoprotection to be achieved in a shorter time, and at lower costs, so there is a rising tendency in health care to use shorter protocols worldwide, including in Poland [22]. It is also difficult to explain why a shorter duration of the initial phase of treatment may increase the feeling of discomfort expressed as being troubled by looking for insects in different situations. It may suggest that this subscale covers a broader sense of burden of treatment and disease itself, but also the necessity of improving the medical information on advantages of shorter protocols. A similar relationship between HRQoL, symptoms of disease and specificity of treatment was also observed in asthmatic patients [23].

In conclusion, we observed the following significant relationships: impact of anxiety upon caution, impact of caution upon limitations level, and impact of both caution and limitations upon discomfort. These results partly resemble the results of the study on the HRQoL of children with HVA treated with VIT and their parents, which showed a significant impact of anxiety on caution in children and their parents, whereas in their parents an additional significant relationship was observed between anxiety and limitations imposed by them on their children, and between parental caution and discomfort [21]. 
An observed decrease in the level of anxiety along with VIT duration needs to be confirmed in follow-up studies, to verify whether the observed relationship reflects real improvement in HRQoL or only a response shift effect. This result also suggests that anxiety seems to be the most important dimension of HRQoL in adolescents treated with VIT.

We did not observe any differences in HRQoL between patients treated against bee and wasp stings, indicating that the tool designed for vespid allergic patients turned out to be useful regardless of the kind of sensitization. This supports the conclusion that problems experienced by all HVA patients, irrespective of the kind of culprit insect, have a lot in common.

The study showed an inverse relationship of borderline significance between VIT duration and level of anxiety, suggesting that VIT may improve HRQoL in some dimensions. However, the crosssectional design of the study does not let us make such an interpretation. Differences between adolescents and children, concerning factors influencing particular dimensions of HRQoL, may reflect real differences concerning the behavioral or social context of their situation (healthy parents of affected children vs. affected children or adolescents), but also may be biased by different characteristics of the adapted scales used in both studies (factor structure, reliability, scale ranges and distribution, sensitivity for different confounding factors).

Previous studies of HRQoL in patients with HVA are limited to adults. The results of studies conducted in adolescents with food allergy suggest that this age group may differ from others with regard to risk-taking and coping strategies [24]. The estimation of a serious decrease in HRQoL due to venom allergy may justify the introduction of specific venom immunotherapy in certain patients with less severe systemic reactions (urticaria, angioedema). Up till now, the decision about the VIT protocol has depended only on medical factors [25]. The present results confirm the need to conduct such studies and use of such sophisticated tools for measuring HRQoL in this group of patients.

In conclusion, place of residence influences HRQoL in Hymenoptera allergic adolescents. Severity of anaphylactic reaction is an independent determinant of anxiety level in adolescents treated with VIT. The VIT protocol influences HRQoL of treated patients.

\section{Acknowledgments}

Supported by a research grant from the Polish Ministry of Science and Higher Education (registration number N N407 254 134). We declare no financial relationship with biotechnology/pharma- ceutical manufacturers mentioned in this manuscript.

\section{References}

1. Bilo BM, Rueff F, Mosbech $\mathrm{H}$, et al. Diagnosis of Hymenoptera venom allergy. Allergy 2005; 60: 1339-49.

2. Bonifazi F, Jutel M, Bilo BM, Birnbaum J, Muller U. Prevention and treatment of hymenoptera venom allergy: guidelines for clinical practice. Allergy 2005; 60: 1459-70.

3. Connolly MA, Johnson JA. Measuring quality of life in paediatric patients. Pharmacoeconomics 1999; 16: 605-25.

4. Juniper EF, Guyatt GH, Dolovich J. Assessment of quality of life in adolescent with allergic rhinoconjunctivitis: developing and testing questionnaire for clinical trials. J Allergy Clin Immunol 1994; 93: 413-23.

5. Varni JW, Limbers CA, Burwinkle TM. How young can children reliably and validly self-report their health-related quality of life? An analysis of 8,591 children across age subgroups with the PedsQL ${ }^{\text {TM }} 4.0$ Generic Core Scales. Health Qual Life Outcomes 2007; 5: 1.

6. Matza LS, Swensen AR, Flood EM, Secnik K, Kline Leidy $N$. Assessment of health-related quality of life in children: a review of conceptual, methodological, and regulatory issues. Value in Health 2004; 7: 79-92.

7. Juniper EF, Guyatt GH, Feeny DH, Ferrie PJ, Griffith LE, Townsend M. Measuring quality of life in children with asthma. Qol Life Res 1996; 5: 35-46.

8. Juniper EF, Howland WC, Roberts NB, Thompson AK, King DR. Measuring quality of life in children with rhinoconjunctivitis. J Allergy Clin Immunol 1998; 101: 163-70.

9. Flokstra-de Blok BMJ, DunnGalvin A, Vlieg-Boerstra BJ, et al. Development and validation of a self-administered Food Allergy Quality of Life Questionnaire for children. Clin Exp Allergy 2008; 39: 127-37.

10. Ware JE, Snow KK, Kosinski M, Gandek B. SF-36 health survey: manual and interpretation guide. The Health Institute, New England Medical Center, Boston 1993.

11. Juniper EF, O'Byrne PM, Guyatt GH, et al. Development and validation of a questionnaire to measure asthma control. Eur Respir J 1999; 14: 902-7.

12. Spielberger CD, Gorsuch RL, Lushene RE. Manual for The State-Trait Anxiety Inventory. Consulting Psychologists Press, Palo Alto, California 1970.

13. Studenski R. Risk and taking risk. Wydawnictwo Uniwersytetu Śląskiego, Katowice 2004.

14. Oude-Elberink JNG, de Konchy JGR, Golden DBK, Brouwer JLP, Guyatt GH, Dubois AEJ. Development and validation of a health-related quality-of-life questionnaire in patients with yellow jacket allergy. J Allergy Clin Immunol 2002; 109: 162-70.

15. Brzyski P, Cichocka-Jarosz E, Tobiasz-Adamczyk B, et al. Theoretical validity and reliability of Vespid Allergy Quality of Life Questionnaire adaptation for Polish adolescents with Hymenoptera venom allergy. Postep Derm Alergol 2012; 29: 8-13.

16. Confino-Cohen R, Melamed S, Goldberg A. Debilitating beliefs, emotional distress and quality of life in patients given immunotherapy for insect sting allergy. Clin Exp Allergy 1999; 29: 1626-31.

17. Hyland ME. The influence of beliefs on the quality of life of patients with allergic diseases. Clin Exp Allergy 1999; 29: 1591-2.

18. Oude-Elberink JNG, Dubois AEJ. Quality of life in insect venom allergic patients. Curr Opin Allergy Clin Immunol 2003; 3: 287-93. 
E. Cichocka-Jarosz, P. Brzyski, E. Świebocka, J. Lange, B. Tobiasz-Adamczyk, G. Lis, U. Jedynak-Wąsowicz, M. Kulus, M. Kaczmarski, T. Małaczyńska, B. Klajna-Kraluk, A. Bręborowicz, Z. Kycler, J.J. Pietrzyk

19. Oude-Elberink JNG, de Konchy JGR, van der Heine S, Guyatt GH, Dubois AEJ. Venom immunotherapy improves health-related quality of life in patients allergic to yellow jacket venom. J Allergy Clin Immunol 2002; 110: 174-82.

20. Brzyski P, Cichocka-Jarosz E, Tobiasz-Adamczyk B, et al. Adaptation of quality of life questionnaires for children with Hymenoptera venom allergy and their parents. Przeg Lek 2010; 67: 1237-42.

21. Cichocka-Jarosz E, Tobiasz-Adamczyk B, Brzyski P, et al. Heath-related quality of life of children with Hymenoptera venom allergy treated with VIT and their parent. Przeg Lek 2010; 67: 1243-8.

22. Cichocka-Jarosz E, Diwakar L, Brzyski P, Tobiasz-Adamczyk B, Lis G, Pietrzyk JJ. Congruence of the current practices in Hymenoptera venom allergic patients in Poland with EAACl guidelines. Arch Med Sci 2011; 7: 832-9.

23. Majewski S, Cichocki P, Stępnicka-Bindemann M, Górski P. Asthma control, quality of life and successful sputum induction. Arch Med Sci 2011; 7: 840-3.

24. Sampson MA, Monoz-Furlong A. Risk-taking and coping strategies of adolescents and young adults with food allergy. J Allergy Clin Immunol 2006; 117: 1440-5.

25. Sturm G, Kraanke B, Rudolph C, Aberer W. Rush Hymenoptera venom immunotherapy: a safe and practical protocol for high-risk patients. J Allergy Clin Immunol 2002; 110: 928-33. 